\title{
Followership im pädagogischen Feld braucht Erfahrung. Ein Vergleich von obligatorischen und berufsbildenden Schulen
}

\section{Kirsten Schweinberger und Brigitte Huber}

Die höhere Gestaltungsfreiheit für die Volksschulen ${ }^{1}$ im Zuge der Teilautonomisierung geht gleichzeitig mit einer grösseren Verantwortung für die Schul- und Unterrichtsqualität einher. Hierbei spielen Lehrpersonen eine zentrale Rolle: Sie müssen in ihrer Rolle als Mitarbeitende Commitment mit den Zielen der Schule und den Entscheidungen der Schulleitung zeigen, aktiv Verantwortung für die Unterrichts- und Schulentwicklung übernehmen sowie stärker im Team kooperieren als es bis anhin üblich war.

Anders als in Volksschulen sind Lehrpersonen an Berufffachschulen stärker mit der Follower-Rolle vertraut. Zum einen ist an Berufsfachschulen bereits seit längerem eine administrativ-organisatorische Führung etabliert, die die pädagogische Leitung, die Personalführung sowie die Schul-und Qualitätsentwicklung verantwortet. Zum anderen verfügen Lehrpersonen an Berufsfachschulen zwingend über einen Stammberuf mit entsprechender Berufserfahrung und sind demnach auch in diesem Zusammenhang bereits mit der Follower-Rolle vertraut.

Der vorliegende Beitrag untersucht, wie sich der unterschiedliche Erfahrungshintergrund von Volks-bzw. Berufffachschullehrpersonen mit der Follower-Rolle auf die selbstberichteten Follower-Attribute einerseits und das Verständnis von guten Mitarbeitenden anderseits auswirkt. Betrachtet werden in diesem Zusammenhang die Attribute Commitment, Aktiver Beitrag für die Schul- und Unterrichtsentwicklung sowie Kooperation im Team.

Befunde zu Gruppenunterschieden in der selbstberichteten Ausprägung an Commitment und Kooperation sowie im Verständnis von guten Mitarbeitenden und deren Implikationen für die Schulpraxis und Forschung werden diskutiert.

Theoretischer Rahmen

Im Zuge ihrer Teilautonomisierung erhalten Volksschulen eine grössere Gestaltungsfreiheit und Eigenverantwortung. In der Deutschschweiz war die "Gewährung von schulischer Teilautonomie systematisch verknüpft mit der 
Implementation von Schulleitungen, die Aufgaben im Bereich der pädagogischen und administrativen Führung der Schule zu übernehmen haben» (Maag Merki \& Steinert, 2006, S. 103). Mit Einführung dieser Hierarchiestufe werden Lehrpersonen verstärkt auch Mitarbeitende. Die neu eingeführten Schulleitungen gelten als treibende Akteure bei der Qualitätssicherung und -entwicklung der Schule: Sie müssen «für Ziele sorgen, organisieren, entscheiden, kontrollieren, messen und beurteilen und die Selbstentwicklung von Menschen fördern» (Bonsen, 2016, S. 311). Vor allem die Zielentwicklung und -setzung werden als zentrale Führungsaufgaben der Schulleitungen verstanden (z.B. Pont, Nusche \& Moorman, 2008; Robinson, Lloyd \& Rowe, 2008). Eine einseitige Fokussierung auf die Schulleitung wird aber der Komplexität des Führungsprozesses nicht gerecht. So argumentieren Litzinger und Schäfer (1982, zitiert nach Baker, 2007) am Beispiel der Zielsetzung, dass Mitarbeitende ihre Unterstützung versagen können, weshalb die Führungsperson Ziele nicht einfach setzen kann, sondern aushandeln muss.

Die Lehrpersonen und ihre Eigenschaften als Follower sind also essentiell für die Erreichung strategischer Ziele und damit für die Schul- und Unterrichtsentwicklung. Der vorliegende Beitrag untersucht, wie der unterschiedliche Erfahrungshintergrund von Volks- bzw. Berufsfachschullehrpersonen mit der Follower-Rolle sich auf die selbstberichteten Follower-Attribute einerseits und das Verständnis von guten Mitarbeitenden anderseits auswirkt.

Der Artikel ist wie folgt aufgebaut: Zunächst wird der theoretische Rahmen dargestellt, wobei das Hauptaugenmerk auf konzeptionellen Ansätze hinsichtlich Followership im Bildungswesen, den Eigenschaften von Lehrpersonen als effektive Follower sowie den Unterschieden zwischen obligatorischen und berufsbildenden Schulen liegt. Der Abschnitt zur Methode behandelt die Stichprobe, die Erhebungsinstrumente und das Vorgehen bei der Analyse. Der Ergebnisteil beinhaltet deskriptive Befunde sowie die Ergebnisse der Hypothesenprüfungen, welche in der Diskussion erörtert werden. Im Rahmen des Fazits wird der Befund zusammengefasst und auf Limitierungen der Studie aufmerksam gemacht.

\section{Followership im Bildungswesen}

Mit der Erweiterung des Konzeptes Schulmanagement (Krüger \& Scheerens, 2012) wird das Verhältnis zwischen der Schulleitung als operative Führung und den Lehrpersonen als Mitarbeitenden in den im Schulkontext verbreiteten Führungstheorien zumindest expliziert. Die transformationale Führung beschränkt sich derweil auf die Identifizierung von Führungsverhalten, welches das Verhalten der Mitarbeitenden zu verändern vermag (Pelz, 2016). Die Einflussnahme erfolgt dabei einseitig von der Führungsperson auf die Mitarbeitenden, diejenige seitens der Lehrpersonen als Mitarbeitende auf die Schulleitung (Followership als «upward leadership», Carsten, Uhl-Bien, West, Patera \& McGregor, 2010, S. 559) wird vernachlässigt. Konzepte der geteilten Führung (wie distributed 
leadership oder teacher leadership) rücken die Lehrperson als aktiven Akteur im Führungsprozess in den Fokus, die Einfluss auf Kolleginnen und Kollegen, die Entscheidungsfindung sowie auf das operative Geschäft nehmen (vgl. Muijs \& Harris, 2003; Poekert, 2012; Robinson, 2009). Diese Einflussnahme kann sich unter anderem in spontaner aufgabenbezogener Zusammenarbeit, in dauerhafter Gremienarbeit wie professionellen Lerngemeinschaften oder durch eine gemeinsame Rollenübernahme vollziehen (Bonsen, 2016). Zwar wird betont, dass die Schulleitung die Bemühungen zur geteilten Führung unterstützen muss (Muijs \& Harris, 2006). Dass eine geteilte Führung aber nur im Rahmen der durch die Schulführung vorgegebenen strategischen Ziele der Schule (vgl. Bonsen, 2016) möglich ist, wird vernachlässigt. In dem per se interaktiven Führungsprozess können Schulleitung und Lehrpersonen in ihren Rollen als operative Führung und Mitarbeitende in ihrer Beziehung zueinander verstanden werden.

Followership ist in diesem Sinne ein Gegenstand der Führungsforschung "[which] refers to the behavior of followers that results from the leader-follower influence relationship» (Densten \& Gray, 2001, S. 70). Bisher erhält das Thema allerdings kaum Aufmerksamkeit (Baker, 2007; Crossman \& Crossman, 2011; Uhl-Bien, Riggio, Lowe \& Carsten, 2014). Demnach ist der Forderung von Densten und Gray (2001) nach einer grösseren Fokussierung auf die Rolle der Mitarbeitenden zuzustimmen - vor allem für den Bereich der Bildungsforschung, wo die Forschungslage zu Followership noch geringer ausfällt (vgl. Clarke, Killeavy \& Ferris, 2015). Lehrpersonen müssen stärkere Beachtung hinsichtlich ihres Beitrags zur Qualitätssicherung und -entwicklung der Schule finden, zumal dieser auch in ihrem Berufsauftrag verankert ist (Departement Bildung, Kultur und Sport, Kanton Aargau, 2006), dies allerdings immer mit Blick auf ihr Verhältnis zur Schulleitung.

Beiträge zu Followership im Bildungswesen beschränken sich auf theoretische Artikel, die die Bedeutung von Followership für die Schulentwicklung betonen (Crippen, 2012a, 2012b; Küpers \& Weibler, 2008; Sergiovanni, 1998) und einzelne empirische Studien, die Aspekte untersuchen wie das Rollenverständnis der Lehrpersonen (Clarke et al., 2015; Mertler, Steyer \& Petersen, 1997, Oktober), Followershipstile (Al-Anshory \& Ali, 2014) oder den Einfluss der Identifikation von Lehrpersonen mit der Schule auf ihr Verhalten und ihre Einstellungen (Van Dick, Hirst, Grojean \& Wieseke, 2007). Wie in der allgemeinen Forschung zu Followership dominiert auch im Bildungsbereich die Konzeption von Kelley (1988) (vgl. Crippen, 2012a; Hinić, Grubor \& Brulić, 2016; Mertler et al., 1997, Oktober). Sein Erhebungsinstrument Followership Style Grid (Kelley, 1992) findet in beiden quantitativen Studien zu Followership im Bildungsbereich Verwendung (Hinić et al., 2016; Mertler et al., 1997, Oktober), obgleich es diesem Instrument an empirischer Fundierung mangelt (vgl. Baker, 2007). 


\section{Schulen als besonderes Umfeld für die Leadership- und Fol lowershipforschung}

Schulen bilden ein besonderes Umfeld in Bezug auf Leader- und Followership: Der zeitlich umfangreichste Tätigkeitsbereich von Lehrpersonen ist das Unterrichten, das dezentralisiert und eigenverantwortlich im Klassenzimmer und in erster Linie in der Interaktion mit ihrer Klasse erfolgt. Die Schulleitung hat daher nur eingeschränkte Möglichkeiten zur Supervision (z.B. classroom walk-through, vgl. Cervone \& Martinez-Miller, 2007; Goldhorn, Kearney \& Webb, 2013, Monat?; Protheroe, 2009). Lehrpersonen nehmen sich in dieser Hinsicht zudem als autonom handelnde Fachexperten einer höchst individualisierten Arbeit wahr, für die die Schule lediglich den strukturellen Rahmen bieten soll (Buhren \& Rolff, 2002). Inwieweit diese Einstellung jedoch mit der Rolle als Follower zu vereinbaren ist, bleibt derzeit unbeantwortet, zumindest lässt sie «Spielräume für suboptimale Tätigkeit» (Altrichter, Rürup \& Schuchart, 2016, S. 109). Dass Lehrpersonen sich mehr als Follower verstehen denn als autonome Fachexperten erfordert eine Führungskultur, die ein positives Bild der Follower-Rolle vermittelt.

\section{Lehrpersonen als effektive Follower}

Gerade die Bildungsreformen der vergangenen Jahre setzen voraus, dass Lehrpersonen sich nicht nur für ihr "Kerngeschäft» Unterricht interessieren, sondern auch aktiv Verantwortung für die Schul- und Unterrichtsentwicklung und damit für die Entwicklung des Gesamtsystems Schule übernehmen (Gruber \& Leutner, 2003; Harazd, Gieske \& Gerick, 2012; Leutwyler \& Herzog, 2010).

Lehrpersonen als effektive Mitarbeitende im Sinne von Follower sollten demnach über Attribute verfügen, die sich als günstige Einflussfaktoren für die Unterrichts- und Schulqualität erwiesen haben. Followership wird damit zu einem ergebnisorientierten Konstrukt: Als positives Follower-Verhalten seitens der Lehrpersonen wird verstanden, was letztlich den Zielen der Schule dient.

\section{Commitment}

Sergiovanni (1992) definiert Follower im Schulkontext als «committed people committed to purposes, a cause, a vision of what the school is and can become, beliefs about teaching and learning, values and standards to which they adhere, and convictions» (S. 71). Auch für Day, Elliot und Kington (2005) gehört die Bindung an Werte zum Commitment dazu; sie erweitern den Begriff aber noch um die Komponenten hohe Standards, kontinuierliche Bemühungen, die eigenen Erfahrungen als Lehrperson zu reflektieren sowie ein intellektuelles wie emotionales Engagement.

Organisationspsychologische Studien betonen schon seit langem die Zusammenhänge zwischen der Bindung einer Person an die Organisation (Commitment) mit Faktoren wie Job Involvement, niedrige Fehlzeiten und in geringerem Masse auch mit der Performanz im Beruf (für Meta-Analysen siehe 
Mathieu \& Zajac, 1990; Riketta, 2002). Hooff und Ridder (2004) konnten zeigen, dass ein höheres Commitment positiv mit der Weitergabe von Wissen innerhalb einer Organisation verbunden ist. Auch für den schulischen Kontext zeigen Studien positive Zusammenhänge zwischen Commitment und Organizational Citizenship Behaviour (Somech \& Bogler, 2002). Andere Arbeiten beschäftigen sich eher mit der Frage, wie das Commitment gestärkt werden kann (Harazd et al., 2012; Henkin \& Holliman, 2009), mit dem Hinweis auf die zu erwartenden positiven Effekte des Commitments auf die Performanz der Lehrpersonen. Als essentielle Bedingung für ein effektives Follower-Verhalten wird Commitment bisher aber im Schulkontext noch nicht diskutiert.

\section{Aktiver Beitrag für die Schul- und Unterrichtsentwicklung}

In der Bildungsforschung werden, wenn von dem Engagement der Lehrpersonen als Mitarbeitende die Rede ist, Begriffe wie distributed leadership oder teacher leadership verwendet (vgl. Poekert, 2012; Robinson, 2009). Bonsen (2016) führt als Übersetzung dazu den Begriff emergente Führung ein, bei der «Individuen kooperieren, um insgesamt die Führungskapazität innerhalb einer Organisation zu erhöhen» (S.317). Fraglich ist, inwieweit es sich dabei tatsächlich um Führungs- oder eher um Followership-Verhalten im Sinne von Uhl-Bien et al. (2014) handelt, die den Führungsprozess explizit als einen gegenseitigen Einfluss von Führungsperson und den Mitarbeitenden beschreiben. Auch Heck und Hallinger (2009) betonen die gegenseitige Beeinflussung bei der geteilten Führung, welche die akademische Leistungsfähigkeit der Schule erhöht, was sich wiederum positiv auf die Leistungsfähigkeit der Schülerinnen und Schüler (in Mathematik) auswirke. Das aktive Einbringen in die Organisation, wie im Konzept teacher leadership vorgesehen, wird allerdings bereits bei Kelley (1988) als Attribut effektiver Follower benannt.

\section{Kooperation im Team}

Die durch Bildungsreformen notwendig gewordene Ausdifferenzierung der Aufgaben für die Lehrpersonen verändert auch ihr Berufsbild (vgl. Holtappels, Lossen, Spillebeen \& Tillmann, 2011; Leutwyler \& Herzog, 2010). Erhöhte Bedeutung erhält in diesem Zusammenhang die Zusammenarbeit im Kollegium. Eine gezielte, gesamtschulische Unterrichtsentwicklung ist nur in enger Kooperation möglich (Buhren \& Rolff, 2002). Zudem wird eine intensivierte Zusammenarbeit im Kollegium auch als Bedingung für professionelles Lernen von Lehrpersonen diskutiert (Bonsen, 2016) sowie mit einer höheren Schulqualität (vgl. Terhart, 2016) in Verbindung gebracht.

Insbesondere im Rahmen der erweiterten Autonomie von Schulen sollen Lehrerkollegien zusammenarbeiten, ein höheres Mass an Verantwortlichkeit für die Ergebnisse ihrer Arbeit übernehmen und das Profil ihrer Schule schärfen (LCH [Dachverband Schweizer Lehrerinnen], 2008, Juni). Allerdings zeigt sich auch, dass die Kooperation von Lehrpersonen noch weiter vorangetrieben 
werden muss, denn «die Berufskultur der Lehrerschaft [ist] noch vielfach vom herkömmlichen Einzelkämpfertum geprägt» (Terhart, 2016, S. 281).

\section{Unterschied zwischen obligatorischen und \\ berufsbildenden Schulen}

In der Schweiz wurde die Einführung der Schulleitung in den obligatorischen Volksschulen als operative Führung, die parallel zur Teilautonomisierung der Volksschulen erfolgte, erst nach der Jahrtausendwende breitenwirksam. Die neu eingerichtete geleitete Schule wird als «in sich geschlossene Organisations- und Betriebseinheit gesehen und erhält mehr Freiräume und Entscheidungskompetenzen» (EDK[Schweizerische Konferenz der kantonalen Erziehungsdirektoren], 2007, S. 26). Die Schulleitungen verantworten die Schul- und Unterrichtsqualität und tragen gegenüber der Schulbehörde Rechenschaft. Die Einführung dieser neuen Hierarchiestufe in den Volksschulen erweitert die Berufsidentität der Lehrpersonen explizit um die Followerrolle. Dies ist anfänglich skeptisch betrachtet worden. So wurden seitens der Lehrpersonen die Einschränkung ihrer Autonomie und die Gefahr einer hohen Machtkonzentration in der Schulleitung befürchtet (Leutwyler, 2003) sowie seitens der Schulleitungen eine fehlende Akzeptanz für deren Führungsfunktion festgestellt (Prince, 2004). Noch 2015 konstatieren Steiner und Landwehr (2015) in dem Monitoringbericht der Externen Schulevaluation des Kantons Aargau, es gäbe noch immer Schulen, «bei denen ein grundsätzlicher Widerstand gegenüber Führung besteht und eine sinnvolle Führungsarbeit extrem schwierig macht» (S. 36). Die vollständige Akzeptanz und Übernahme der Followerrolle seitens der Lehrpersonen scheint also nach wie vor noch nicht abgeschlossen.

Ergeben sich auf der Ebene der Volksschulen noch Entwicklungsprozesse bezüglich der Rollenfindung zwischen Führung und Mitarbeitenden, so übernimmt die Schulleitung auf der Sekundarstufe II bereits «seit Längerem Aufgaben in administrativ-organisatorischen Bereichen, in der pädagogischen Leitung, bei der Personalführung sowie in der Schul- und Qualitätsentwicklung» (EDK, 2007, S. 27). Berufsfachschulen beispielsweise sind in der Regel hierarchisch gegliedert und werden von einem Schulleiter beziehungsweise bei grösseren Schulen von einem Leitungsteam mit Abteilungsleitenden oder Fachvorstehenden geführt. Darüber hinaus haben Berufsfachschullehrpersonen, anders als Volksschullehrpersonen, zwingend einen Beruf erlernt, in dem sie den höchsten Bildungsabschluss in ihrer Berufskategorie erworben haben und zudem eine gewisse Anzahl Jahre Berufserfahrung ausweisen müssen, um Berufsfachschullehrperson werden zu können (vgl. Hof, Strupler \& Wolter, 2011). Berufsfachlehrpersonen verfügen also bereits vor ihrer Lehrkarriere über eine Berufserfahrung als Lernende und Mitarbeitende und somit über Erfahrung in der Follower-Rolle. Sie sind dann als Lehrpersonen in einem schulischen Umfeld tätig, in welchem die hierarchische Gliederung in Schulleitung und Mitarbeitende und folglich die Follower-Rolle bereits etabliert sind. 
Volksschul- und Berufsfachlehrpersonen unterscheiden sich demnach in der Länge und wahrscheinlich auch Breite ihrer Erfahrung als Follower. Daraus resultieren die im Folgenden dargestellten Hypothesen.

\section{Hypothesen}

Die Teilautonomisierung der Schulen und die damit verbundene Einführung einer operativen und strategischen Schulführung führen zu neuen Anforderungen an Lehrpersonen. Wo sie früher als autonom handelnde Fachexperten ihren Beruf erfüllen konnten, sind heute auch Attribute wie Commitment und Kooperation gefragt. Dass Lehrpersonen von Berufsfachschulen in diesem Prozess aufgrund einer längeren Führungstradition sowie der zwingenden Berufserfahrung in ihrem Stammberuf weiter fortgeschritten sind als Lehrpersonen von Volksschulen, zeigt sich in ihren höheren Ausprägungen hinsichtlich der untersuchten Followership-Aspekte Commitment, dem Beitrag zur Schulund Unterrichtsentwicklung sowie bezüglich der berichteten Kooperation:

H1: Im Bereich des Commitments zeigen Lehrpersonen von Berufsfachschulen höhere Werte als Lehrpersonen von Volksschulen.

H2: Im Bereich der Kooperation zeigen Lehrpersonen von Berufsfachschulen höhere Werte als Lehrpersonen von Volksschulen.

H3: Bezüglich des aktiven Beitrages zur Schul- und Unterrichtsentwicklung zeigen Lehrpersonen von Berufsfachschulen höhere Werte als Lehrpersonen von Volksschulen.

Ergänzend soll untersucht werden, ob sich die proklamierten Unterschiede in den befragten Followershipdimensionen auch in einem Unterschied bezüglich der Passung zwischen der eigenen Person und dem idealen Mitarbeitenden manifestiert.

Die Unterschiede zwischen Volksschul- und Berufsfachlehrpersonen hinsichtlich der Länge und wahrscheinlich auch Breite ihrer Erfahrung als Follower zeigt sich darüber hinaus in einem unterschiedlichen Verständnis von guten Mitarbeitenden. Folglich wird vermutet:

H4: Es zeigen sich in beiden Gruppen unterschiedliche Zusammenhänge zwischen den Followershipdimensionen und der Selbsteinschätzung zur eigenen Rolle als Mitarbeitende(r).

\section{Methode}

\section{St ichprobe}

Im Zuge einer Online-Untersuchung wurden 309 Lehrpersonen aus dem Nordwestschweizer Bildungsraum befragt, darunter 105 Volksschul- und 204 Berufsfachschullehrpersonen. 
Die obligatorische Volksschule in der Schweiz umfasst die Schulstufen Kindergarten, Primarschule sowie die Sekundarstufe 1. In der vorliegenden Stichprobe der Volksschullehrpersonen waren 17\% Kindergarten-, 52\% Primarschullehrpersonen und die übrigen 30\% Lehrpersonen auf der Sekundarstufe 1.

Alter und Betriebszugehörigkeit wurde auf ordinalem Skalenniveau erfasst (vgl. Tabelle 1). Die befragten Volksschullehrpersonen und Lehrpersonen der Berufsfachschulen unterscheiden sich nicht hinsichtlich der Altersstruktur $\left(\chi^{2}(3)\right.$ $=4.09, \mathrm{p}=.252)$, der Betriebszugehörigkeit zur Schule $\left(\chi^{2}(3)=6.54, \mathrm{p}=.088\right)$ und des Pensenumfangs $\left(\chi^{2}(2)=4.39, \mathrm{p}=.111\right)$. In beiden Gruppen fallen die meisten Lehrpersonen in die Altersgruppen 41 - 50 bzw. über 50 Jahre (73\% im Bereich der Volksschule vs. 70\% im Bereich der Berufsfachschulen), sind bereits mehr als fünf Jahre an der betreffenden Schule tätig (59\% im Bereich der Volksschule vs. 68\% im Bereich der Berufsfachschulen) und mit einem Pensum zwischen 71 und 100\% angestellt (52\% im Bereich der Volksschule vs. 55\% im Bereich der Berufsfachschulen).

Tabelle 1: Alter und Betriebszugehörigkeit zur Schule nach Schultyp

\begin{tabular}{llccc} 
& & $\begin{array}{c}\text { Volks- } \\
\text { schule }\end{array}$ & $\begin{array}{c}\text { Berufsfach- } \\
\text { schule }\end{array}$ & Gesamt \\
\hline Altersgruppen & 30 oder jünger & 11 & 14 & 25 \\
& zwischen 31 und 40 & 17 & 46 & 63 \\
& zwischen 41 und 50 & 29 & 67 & 96 \\
& über 50 & 47 & 76 & 123 \\
& Total & 104 & 203 & 307 \\
\hline Betriebszugehörigkeit & weniger als ein Jahr & 14 & 12 & 26 \\
& zwischen 1 und 3 Jahren & 14 & 32 & 46 \\
& zwischen 3 und 5 Jahren & 15 & 22 & 37 \\
& mehr als 5 Jahre & 61 & 138 & 199 \\
& Total & 104 & 204 & 308 \\
\hline
\end{tabular}

Ein signifikanter Unterschied zwischen beiden Gruppen besteht in der Geschlechterverteilung $\left(\chi^{2}(1)=88.26, \mathrm{p}<.001\right)$. In der Gesamtstichprobe sind bei drei fehlenden Werten 139 Frauen (45\%) und 167 Männer (54\%) vertreten. In der Gruppe der Volksschullehrpersonen liegt der Frauenanteil bei 83\%, in der Gruppe der Berufsfachschullehrpersonen lediglich bei $26 \%$. 


\section{Erhebungsinstrumente}

\section{Follower-Verhalten bei Lehrpersonen}

Die Forderung Bakers (2007) nach reliablen und validen Erhebungsinstrumenten von Followership erhält noch stärkere Dringlichkeit im Kontext der Schulführungsforschung, die erst im Zuge der Implementierung geleiteter Schulen das Verhältnis von Lehrpersonen und Schulleitung in den Begrifflichkeiten von Leader und Follower thematisierte. Gemäss der Kritik Bakers (2007) an der unzureichenden empirischen Grundlage sowie die aus früheren Studien hervorgehende, unzureichende Trennschärfe bei der Typenzuordnung im pädagogischen Bereich (Hinić et al., 2016; Mertler et al., 1997, Oktober), entwickelten Schweinberger und Huber (in Druck) ein Fragebogenverfahren zur Erfassung des Verhaltens von Lehrpersonen in ihrer Rolle als Mitarbeitende (vgl. Tabelle 2).

\section{Tabelle 2: Items des eingesetzten Fragebogenverfahrens}

\begin{tabular}{|c|c|}
\hline Skala & Items \\
\hline \multirow[t]{3}{*}{ Commitment } & $\begin{array}{l}\text { Ich versuche, den Anforderungen, die die Schulleitung an mich als } \\
\text { Mitarbeitende/n stellt, gerecht zu werden. }\end{array}$ \\
\hline & Ich trage die langfristigen Ziele unserer Schulführung mit. \\
\hline & Die Rückmeldung der Schulleitung nehme ich ernst. \\
\hline \multirow{3}{*}{$\begin{array}{l}\text { Aktiver Beitrag für } \\
\text { die Schul- und Unter- } \\
\text { richtsentwicklung }\end{array}$} & $\begin{array}{l}\text { Ich setze mich gern mit neuen Ideen zur Schul- und } \\
\text { Unterichtsentwicklung auseinander. }\end{array}$ \\
\hline & $\begin{array}{l}\text { Wenn unsere Schulleitung neue Ideen zur Schul- und } \\
\text { Unterichtsentwicklung vorstellt, setze ich mich aktiv damit auseinander. }\end{array}$ \\
\hline & $\begin{array}{l}\text { Bei Arbeiten in Schulentwicklungsprojekten (z.B. Arbeit in Steuergruppen, } \\
\text { Unterrichtsteams, Q-Gruppen) trage ich gern meinen Teil bei. }\end{array}$ \\
\hline \multirow[t]{3}{*}{ Kooperation im Team } & $\begin{array}{l}\text { Den Lehrpersonen unserer Schule ist es wichtig, dass eine kooperations- } \\
\text { förderliche Gemeinschaft entstehen kann. }\end{array}$ \\
\hline & $\begin{array}{l}\text { Die Lehrpersonen unserer Schule kehren Konflikte und Probleme nicht } \\
\text { unter den Teppich, sondern sprechen sie offen an. }\end{array}$ \\
\hline & $\begin{array}{l}\text { Ich bin zufrieden damit, wie sich das Kollegium im Entscheidungs- } \\
\text { prozess verhält. }\end{array}$ \\
\hline
\end{tabular}

Der dreidimensionale Fragebogen umfasst die Skalen Commitment, Aktiver Beitrag für die Schul- und Unterrichtsentwicklung sowie Kooperation im Team und greift damit Aspekte auf, die sich sowohl als positive Eigenschaften von Mitarbeitenden allgemein als auch als günstige Einflussfaktoren auf eine gelingende Schulentwicklung erwiesen haben. Den Befragten wurden Aussagen präsentiert, zu der sie ihre Zustimmung auf einer sechsstufigen Likertskala ausdrücken können. Höhere Werte bedeuten eine höhere Ausprägung auf der entsprechenden Skala. 
Das Instrument ist sehr ökonomisch und reliabel. Auch erste Belege für die Konstruktvalidität, welche die Interpretation der Testergebnisse im Sinne des zugrundeliegenden Konzeptes erlaubt, konnten erbracht werden (Schweinberger \& Huber, in Druck). Die Skalendokumentation ist der Tabelle 3, die Interkorrelationen der Followership-Skalen sind der Tabelle 4 zu entnehmen.

\section{Tabelle 3: Deskriptive Statistik und Interne Konsistenzen (Cronbachs Alpha) der eingesetzten Skalen}

\begin{tabular}{llccccc}
\hline & & N & MW & SD & $\mathrm{N}_{\text {items }}$ & $\begin{array}{c}\text { Cron- } \\
\text { bachs a }\end{array}$ \\
\hline $\begin{array}{l}\text { Followership bei } \\
\text { Lehrpersonen }\end{array}$ & $\begin{array}{l}\text { Commitment }^{\mathrm{a}} \\
\text { Aktiver Beitrag für } \\
\text { die Schul- und Unter- } \\
\text { richtsentwicklung }\end{array}$ & 271 & 5.28 & 0.69 & 3 & .76 \\
& Kooperation im Team $^{\mathrm{a}}$ & 239 & 4.82 & 0.91 & 3 & .78 \\
\hline $\begin{array}{l}\text { Passung von Ideal } \\
\text { und Real }\end{array}$ & V-ILT $^{1}$ & 292 & 4.68 & 1.60 & 1 & -* $^{*}$ \\
& V-IFT $^{2}$ & 293 & 5.36 & 0.95 & 1 & - $^{*}$ \\
\hline
\end{tabular}

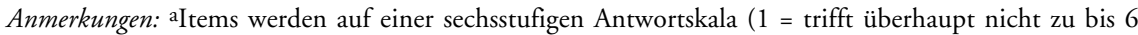
= trifft vollkommen zu) beantwortet; höhere Werte bedeuten eine höhere Ausprägung auf der entsprechenden Skala. ${ }^{1}$ Venn-Diagramm als Mass der Passung zwischen der realen Schulleitung und dem impliziten idiosynkratischen Schulleitungsprototyp, ${ }^{2}$ Venn-Diagramm als Mass der Passung zwischen der eigenen Person und dem impliziten idiosynkratischen Mitarbeitendenprototyp, adaptiert nach van Quaquebeke und Brodbeck (2008); *Berechnung bei Ein-Item-Konstruktionen nicht möglich

\section{Tabelle 4: Interkorrelationen der Followership-Skalen}

\begin{tabular}{lccc}
\hline & Commitment $^{a}$ & $\begin{array}{c}\text { Aktiver Beitrag fir } \\
\text { die Schul- und Unter- } \\
\text { richtsentwicklung }\end{array}$ & Kooperation im Team \\
\hline $\begin{array}{l}\text { Commitment } \\
\text { Aktiver Beitrag für die Schul- } \\
\text { und Unterrichtsentwicklung }\end{array}$ & $.45^{* * *}$ & $.39^{* * *}$ \\
Kooperation im Team & - & - & $.17^{*}$ \\
\hline
\end{tabular}

Anmerkungen: aItems werden auf einer sechsstufigen Antwortskala $(1=$ trifft überhaupt nicht zu bis $6=$ trifft vollkommen zu) beantwortet; $\mathrm{N}=197 ;^{*} \mathrm{p}<.05 ;{ }^{* *} \mathrm{p}<.01 ;{ }^{* * *} \mathrm{p}<.001$

\section{Erfassung der Passung von Ideal und Real bei Schulleitung und Lehrpersonen} Der V-ILT (van Quaquebeke \& Brodbeck, 2008) ist ein «piktorales Mass zur Erhebung der Passung mit einem idiosynkratischen Führungsprototyp» (S. 70). Mit Hilfe eines Venn-Diagramms werden die Befragten gebeten, die Passung zwischen ihrer Führungsperson und ihrer Vorstellung einer idealen Führungsperson durch den Grad der Überschneidung anzugeben. Dabei werden sieben Abstufungen der zunehmenden Überschneidung visualisiert, bei der Stufe 
sieben wird Deckungsgleichheit erreicht. Die grafische Darstellung ermöglicht den «Verzicht auf eine konkrete inhaltliche Ausgestaltung» (van Quaquebeke \& Brodbeck, 2008, S. 77) und überlässt es den Befragten, die für sie «relevanten Attribute eines idealen Führungsprototyps» (van Quaquebeke \& Brodbeck, 2008, S. 75) sowie in der Anpassung auch Mitarbeitendenprototyps zu projizieren.

Für die vorliegende Untersuchung wurde das Verfahren angepasst. Die befragten Lehrpersonen wurden nun einmal gebeten die Passung zwischen ihrer realen Schulleitung und ihrer idealen Schulleitung (V-ILT) und zum zweiten auch die Passung ihrer eigenen Person als Mitarbeitende mit einem idealen Mitarbeitenden (V-IFT) einzuschätzen (vgl. Tabelle 3).

\section{Ergebnisse}

Um die Unterschiede zwischen Berufsfachschullehrpersonen und Volksschullehrpersonen hinsichtlich ihrer Ausprägung bezüglich der vorgestellten Followership-Aspekte zu untersuchen, wurde eine Multivariate Varianzanalyse gerechnet. Dabei wurde die Zugehörigkeit zur Berufsfach- vs. Volksschule als unabhängige, die drei Variablen Commitment, aktiver Beitrag zur Schul- und Unterrichtsentwicklung sowie Kooperation im Team als abhängige Variablen in die Analyse eingebracht. Um den signifikanten Unterschied hinsichtlich der Geschlechterverteilung zu berücksichtigen, wurde das Geschlecht als Kovariate eingeführt. Während der Geschlechtseffekt nicht signifikant wird $(F(3,190)=$ $1.63, \mathrm{p}=.185$, Wilk's Lambda=.98), erreicht der Effekt des Schultyps statistische Signifikanz $(\mathrm{F}(3,190)=7.61, \mathrm{p}<.001$, Wilk's Lambda=.89).

Um herauszufinden, auf welcher bzw. welchen Dimensionen sich der multivariate Unterschied manifestiert, wurden drei t-Tests mit Bonferroni-Korrektur (angepasstes $\alpha=.017$ ) und paarweisem Ausschluss angeschlossen. Dabei zeigten sich erwartungsgemäss signifikante Unterschiede sowohl bezüglich Commitment $(t(269)=-2.87, p=.005$, Cohen's $d=0.37)$ als auch hinsichtlich der Kooperation im Team $(t(123.693)=-4.04, p<.001$, Cohen's $d=0.61)$. Auf beiden Dimensionen zeigen Berufsfachschullehrpersonen durchschnittlich höhere Werte (vgl. Tabelle 5). Keine Unterschiede liessen sich, wider Erwarten, bezüglich des aktiven Beitrages zur Schul- und Unterrichtsentwicklung finden $(t(258)=-0.66, p=.509$, Cohen's $d=0.09$ ).

$\mathrm{Ob}$ sich die Unterschiede in den befragten Followershipdimensionen auch in einem Unterschied bezüglich der Passung zwischen der eigenen Person und dem idealen Mitarbeitenden manifestiert, wurde mittels eines weiteren t-Tests ermittelt. Hier zeigt sich ein Mittelwertsunterschied von kleiner bis mittlerer Effektstärke in erwarteter Richtung, $t(216.112)=-3.91, p<.001$, Cohen's $d=0.47$. Berufsfachschullehrpersonen berichten einen höheren Passungsgrad zwischen der eigenen Person und dem impliziten idiosynkratischen Mitarbeitendenprototyp als Volksschullehrpersonen. 
Tabelle 5: Deskriptive Statistik für die drei Followershipdimensionen, getrennt für Volksschul- und Berufsfachlehrpersonen

\begin{tabular}{llccc}
\hline & & N & Mittelwert & $\begin{array}{c}\text { Standard- } \\
\text { abweichung }\end{array}$ \\
\hline Commitment & Volksschule & 85 & 5.11 & 0.75 \\
& Berufsfachschule & 186 & 5.36 & 0.64 \\
$\begin{array}{l}\text { Aktiver Beitrag zur } \\
\text { Schul- und Unter- }\end{array}$ & Volksschule & 93 & 4.77 & 0.90 \\
richtsentwicklung & Berufsfachschule & 167 & 4.85 & 0.91 \\
Kooperation im Team & Volksschule & 80 & 4.19 & 0.99 \\
& Berufsfachschule & 159 & 4.69 & 0.73 \\
V-IFT & Volksschule & 98 & 5.07 & 0.85 \\
& Berufsfachschule & 195 & 5.50 & 0.96 \\
\hline
\end{tabular}

Anmerkungen: ${ }^{1}$ Venn-Diagramm als Mass der Passung zwischen der eigenen Person und dem impliziten idiosynkratischen Mitarbeitendenprototyp, adaptiert nach van Quaquebeke und Brodbeck (2008)

Inwieweit die präsentierten Followershipdimensionen in Zusammenhang mit der Selbsteinschätzung zum Passungsgrad zwischen der eigenen Person und dem idealen Mitarbeitenden bezüglich der wahrgenommenen Followerrolle stehen und ob sich dabei Gruppenunterschiede ergeben, wurde untersucht, indem die entsprechenden Korrelationen getrennt für beide Gruppen berechnet wurden. Anschliessend wurde überprüft, inwieweit sich die Korrelationen aus den Gruppen voneinander unterscheiden. Die Ergebnisse sind in der Tabelle 6 ersichtlich. In beiden Gruppen korrelieren alle Followershipdimensionen mit der Selbsteinschätzung zum Passungsgrad zwischen der eigenen Person und dem idealen Mitarbeitenden. Allerdings unterscheidet sich die Stärke der Korrelation zwischen Selbsteinschätzung und dem berichteten Commitment: Während sich bei den Berufsfachschullehrpersonen ein starker Zusammenhang zeigt ( $\mathrm{r}=.54$; $\mathrm{R}^{2}=.30$, coefficient of determination oder geteilte Varianz, Field, 2013, S.179) , ist dieser bei den Volksschullehrpersonen klein $(r=.28$; $\mathrm{R} 2=.08$, Field, 2013, S.179).

Auffällig ist zudem, dass sich bei Berufsfachschullehrpersonen ein starker Zusammenhang zwischen der Einschätzung zur Schulleitung und der eigenen, bei Volksschullehrpersonen jedoch kein signifikanter Zusammenhang zeigt. 
Tabelle 6: Korrelationen zwischen den Followershipdimensionen und der Selbsteinschätzung zum Passungsgrad zwischen der eigenen Person und dem idealen Mitarbeitenden

\begin{tabular}{lcclc} 
& \multicolumn{2}{c}{$\begin{array}{c}\text { V-IFT } \\
\text { Volksschule } \\
\mathrm{N}=64\end{array}$} & $\begin{array}{c}\text { Berufsfachschule } \\
\mathrm{N}=129\end{array}$ & $\begin{array}{l}\text { Vergleich beider } \\
\text { Korrelationskoeffizenten }\end{array}$ \\
\hline $\begin{array}{l}\text { Commitment } \\
\begin{array}{l}\text { Aktiver Beitrag für } \\
\text { die Schul- und Unter- } \\
\text { richtsentwicklung }\end{array}\end{array}$ & $.28^{*}$ & $.54^{* * *}$ & $z=-2.03, \quad p=.021$ \\
$\begin{array}{l}\text { Kooperation im Team } \\
\text { V-ILT1 }\end{array}$ & $.30^{*}$ & $.26^{*}$ & $z=0.31, \quad p=.380$ \\
\hline
\end{tabular}

Anmerkungen: ${ }^{1}$ Venn-Diagramm als Mass der Passung zwischen der realen Schulleitung und dem impliziten idiosynkratischen Schulleitungsprototyp, 2Venn-Diagramm als Mass der Passung zwischen der eigenen Person und dem impliziten idiosynkratischen Mitarbeitendenprototyp, adaptiert nach van Quaquebeke und Brodbeck (2008); ${ }^{*} \mathrm{p}<.05 ;{ }^{* *} \mathrm{p}<.01$; $^{* * *} \mathrm{p}<.001$

\section{Diskussion}

Der vorliegende Beitrag untersucht, wie die unterschiedliche Führungstradition in der Volks- bzw. Berufsfachschule sowie die vorgängigen Erfahrungen der Berufsfachschullehrpersonen als Follower, sich auf die selbstberichteten Follower-Attribute einerseits und das Verständnis von guten Mitarbeitenden anderseits auswirkt.

Dabei zeigen sich Gruppenunterschiede in der selbstberichteten Ausprägung an Commitment und Kooperation im Team. In beiden Dimensionen weisen Lehrpersonen der Volksschulen geringere Werte auf als Lehrpersonen von Berufsfachschulen. Ist der Effekt bei Commitment noch gering, so doch bezüglich der Kooperation von mittlerer Grösse. Wider Erwarten ergeben sich keine signifikanten Unterschiede hinsichtlich des aktiven Beitrages zur Schul- und Unterrichtsentwicklung.

Die nur geringen Unterschiede hinsichtlich der Skala Commitment können als Hinweis gedeutet werden, dass die neu eingerichtete geleitete Schule und die damit einhergehende Einführung der Schulleitungen inzwischen seitens der Volksschullehrpersonen höhere Akzeptanz erfahren. Dies wird durch andere Untersuchungen durchaus gestützt (Büeler \& Imgrüth, 2003; Steger Vogt \& Appius, 2011). Für die Berufsfachschullehrpersonen stellt das Commitment das stärkste Korrelat der Passung zwischen der eigenen Person und dem impliziten idiosynkratischen Mitarbeitendenprototyp dar - dies mit einer geteilten Varianz von $30 \%\left(\mathrm{R}^{2}=.30\right)$. Für sie ist folglich das Commitment wesentlicher Bestandteil eines idealen Mitarbeitenden. Überraschend ist, dass dies für Volksschullehrpersonen nicht zutrifft. Für die Volksschullehrpersonen zeigt sich hier der schwächste Zusammenhang mit einer geteilten Varianz von $8 \%\left(\mathrm{R}^{2}=.08\right)$. 
Ein starkes Commitment ist demnach für Volksschullehrpersonen ein deutlich weniger wichtiger Aspekt eines idealen Mitarbeitenden. Dieses Ergebnis ist besonders bemerkenswert, da - bezogen auf den Schulkontext - Commitment gemäss Sergiovanni (1992) das definierende Element innerhalb des Konzepts Followership darstellt und auch Kelley (1988) ausführt, dass sich herausragende Mitarbeitende einem Produkt, einem (Organisations-) Ziel bzw. einer Organisation verpflichtet fühlen.

Beachtung sollte ferner der nicht unerhebliche Unterschied im selbstberichteten Kooperationsverhalten zwischen Lehrpersonen von Berufsfachschulen sowie Lehrpersonen der Volksschulen finden - dies vor allem vor dem Hintergrund der gestiegenen Bedeutung von Kooperation für die Schul- und Unterrichtsqualität (Holtappels et al., 2011; Steinert \& Maag Merki, 2009; Terhart \& Klieme, 2006). Insbesondere im Zuge der erweiterten Autonomie von Schulen sind Lehrpersonen aufgefordert, verstärkt Verantwortung für die Gesamtschule zu übernehmen, was nur mittels intensiverer Kooperation möglich ist. Hier zeigt sich einerseits auch in der vorliegenden Untersuchung, dass die Kooperation von Lehrpersonen noch weiter vorangetrieben werden muss (vgl. Terhart, 2016; Terhart \& Klieme, 2006). Andererseits deutet der mittlere Zusammenhang zwischen der Skala Kooperation und der Passung zwischen der eigenen Person und dem impliziten idiosynkratischen Mitarbeitendenprototyp darauf hin, dass sich auch bei Volksschullehrpersonen das Verständnis durchsetzt, dass Kooperation einen wichtigen Aspekt im Follower-Verhalten darstellt. Hier gilt es anzusetzen, um Kooperationsgelegenheiten zu ermöglichen.

Kein Unterschied zwischen Lehrpersonen der Berufsfachschulen und der Volksschulen zeigte sich wider Erwarten hinsichtlich der Skala aktiver Beitrag zur Schul- und Unterrichtsentwicklung. Wenngleich davon auszugehen ist, dass eine gezielte gesamtschulische Unterrichtsentwicklung nur in enger Kooperation (Buhren \& Rolff, 2002) und durch die koordinierende Instanz der Schulleitung (Bonsen, 2016) möglich ist, so fühlten sich Lehrpersonen auch vor der Teilautonomisierung der Schulen für die Qualität ihres Unterrichts verantwortlich (z.B. Eikenbusch, 2002). Dieser Aspekt des Follower-Verhaltens ist einerseits inhaltlich eng mit dem zentralen Tätigkeitsbereich von Lehrpersonen verknüpft und andererseits durch die Betonung der Eigenverantwortlichkeit mit der Eigenwahrnehmung als autonom handelnde Fachexperten (Buhren \& Rolff, 2002) konform. Die neue Rolle der Lehrperson als Follower zeigt sich dann eher darin, dass die Steuerung der Unterrichts- und Schulqualität durch die Schulleitung akzeptiert wird (Skala Commitment) und dass der aktive Beitrag sich nun auch in partnerschaftlichen Beziehungen zwischen Kollegen (Skala Kooperation im Team) manifestiert, um die Lehr- und Lernpraktiken und damit die Unterrichtsqualität zu verbessern (Poekert, 2012).

Ein weiterer zu diskutierender Befund ist die unterschiedliche Stärke des Zusammenhangs zwischen der Passung der eigenen Person und dem impliziten idiosynkratischen Prototyp eines Mitarbeitenden einerseits und der Passung 
zwischen der realen Schulleitung und dem impliziten idiosynkratischen Schulleitungsprototyp andererseits. Für die Berufsfachschullehrpersonen zeigt sich an dieser Stelle der stärkste Zusammenhang, für Volksschullehrpersonen hingegen der geringste. Das Verständnis, dass die eigene Qualität als Follower massgeblich die Qualität der Schulleitung bedingt und umgekehrt, mag ein Hinweis darauf sein, dass Berufsfachschullehrpersonen Führung als einen dyadisch gestalteten Prozess verstehen. Dies unterscheidet sie deutlich von den Volksschullehrpersonen, die die eigene Qualität als Follower als eher unabhängig von der Qualität der Schulleitung bewerten. Die eingangs kritisierte alleinige Fokussierung auf die Schulleitung scheint partiell auch von den Lehrpersonen geteilt zu werden (siehe auch Schweinberger \& Huber, in Druck).

Im Folgenden sollen Limitationen der Studie und deren Adressierung in künftigen Forschungsbemühungen diskutiert werden. In diesem Beitrag wird davon ausgegangen, dass die unterschiedliche Erfahrung mit dem GeführtWerden ursächlich für die Gruppenunterschiede zwischen Berufsfachschullehrpersonen und Volksschullehrpersonen ist. Untersucht wurden aber natürliche Gruppen; der tatsächliche Grössenunterschied an Followership-Erfahrung kann nicht quantifiziert werden. Es ist anzunehmen, dass die unterschiedliche Erfahrungsbreite und -länge als Follower massgeblich zu den gefundenen Unterschieden beiträgt; andere Einflüsse können aber nicht ausgeschlossen werden. Quereinsteigende in den Lehrberuf an Volksschulen haben ähnlich wie Berufsfachschullehrpersonen einen Stammberuf und folglich mit hoher Wahrscheinlichkeit bereits Erfahrung darin, geführt zu werden. Ihre Einschätzungen zu den untersuchten Followershipdimensionen könnten weiter Aufschluss darüber geben, welchen Einfluss die unterschiedliche Erfahrung als Follower auf die Wahrnehmung der Rolle hat.

Die in Anlehnung an van Quaquebeke und Brodbeck (2008) verwendeten V-IFT- und V-ILT- Diagramme sind piktorale Masse, um die Passung mit einem idiosynkratischen Prototypen, entweder dem eines Mitarbeitenden (IFT) oder dem einer Führungsperson (ILT) zu erheben. Dieses Verfahren verspricht eine hohe Effizienz und Kulturunabhängigkeit (van Quaquebeke \& Brodbeck, 2008) sowie eine hohe Anschaulichkeit für die Befragten bei der Einschätzung. Offen bleibt jedoch der inhaltliche Gehalt des individuell herangezogenen Prototyps. Die Zusammenhänge zwischen den untersuchten Followership-Dimensionen und der Einschätzung zur Passung zeigen, dass sie wichtige Indikatoren eines idealen Mitarbeitenden sind. Künftige Forschungsbemühungen sollten die Attribute, die Lehrpersonen mit einer idealen Schulleitung beziehungsweise einem idealen Mitarbeitenden im Schulkontext verbinden, noch weiter explizieren. In einem weiteren Schritt können dann auch Zusammenhänge zwischen dem Erfahrungshintergrund als Mitarbeitende und den Attributen des idiosynkratischen Prototyps beleuchtet werden. Die in diesem Beitrag verwendeten Selbstberichte sollten zudem um objektivere Masse des Follower-Handelns erweitert werden. 


\section{Fazit und Implikationen}

Lehrpersonen spielen eine zentrale Rolle für die Unterrichts- und Schulqualität (Harazd et al., 2012; Leutwyler \& Herzog, 2010). Ihr Widerstand kann die Umsetzung von Entwicklungsmassnahmen erschweren oder gar verunmöglichen. Umso wichtiger scheint es zu klären, wie Lehrpersonen ihre Rolle als Mitarbeitende im Führungsprozess verstehen. Die Befunde der vorliegenden Studie lassen vermuten, dass eine grössere Erfahrung und Routine im Umgang mit dem Geführt-Werden zu einem veränderten Bild der Follower-Rolle führt. Für Volksschulen mögen ein offener Austausch unter den Lehrpersonen sowie die klare Erwartungsformulierung seitens der Schulleitungen helfen, diesen Prozess $\mathrm{zu}$ beschleunigen. Dies betrifft insbesondere die Akzeptanz, dass die eigenen Bemühungen bezüglich der Schul- und Unterrichtsentwicklung einhergehen mit dem Commitment gegenüber den strategischen Entscheidungen der Schulführung sowie einer verstärkten Auseinandersetzung und Zusammenarbeit im Kollegium.

Das Verständnis von Volksschullehrpersonen als Mitarbeitende ist nicht nur in der Schulpraxis neu, auch in der Forschung ist die Perspektive noch unterrepräsentiert. So erfolgte beispielsweise im Rahmen einer Bestandesaufnahme zur externen Schulevaluation in der Schweiz die Bewertung des Führungsprozesses ausschliesslich durch die Beurteilung des Schulführungshandelns; das Verhalten der Lehrpersonen blieb unberücksichtigt (vgl. Mahler \& Quesel, 2015). Das Verständnis, dass sich der Führungsprozess in der Wechselwirkung zwischen Schulleitung und Lehrpersonen vollzieht, muss sich bei den Akteuren noch durchsetzen. Erst dann werden beispielsweise professionelle Lerngemeinschaften, wie sie derzeit als «Königsweg für Qualitätsverbesserungen» (Bonsen \& Rolff, 2006, S. 167) diskutiert werden, möglich. Diese setzen ein erhöhtes Verantwortungsbewusstsein der Lehrperson für die Gesamtschule und nicht nur für den eigenen Unterricht und die eigene Klasse voraus.

Eine stärkere Einbindung der Follower-Perspektive in die Forschung zu Unterrichts- und Schulqualität kann nicht nur das Verständnis für die Bedeutung der Lehrpersonen im Wirkprozess der Schulentwicklung erhöhen, sondern auch den theoretischen Rahmen für die vertiefte Auseinandersetzung mit dem Zusammenspiel von Schulleitung und Kollegium bei der Etablierung einer gelingenden Schulentwicklung erweitern. Interventionsstudien zur Entwicklung relevanter Follower-Attribute sind genauso denkbar wie die Analyse der Follower-Eigenschaften von Lehrpersonen hinsichtlich ihres Einflusses auf die Schulqualität und letztlich auf die Leistungen von Schülerinnen und Schülern.

\section{Anmerkung}

1 Die obligatorische Schule in der Schweiz, auch Volksschule, setzt sich aus der Primarstufe und der Sekundarstufe I zusammen. In den meisten Kantonen ist der Besuch des Kindergartens während eines oder zwei Jahren obligatorisch. Die Verantwortung für die obligatorische Schule obliegt den Kantonen. Die Gemeinden organisieren den Schulbetrieb. Im Folgenden wird der in der Schweiz übliche Begriff Volksschule verwendet. 
L'école obligatoire en Suisse, également dit école élémentaire, comprend l'école primaire et l'école secondaire du premier cycle. Dans la plupart des cantons, la fréquentation du jardin d'enfants est obligatoire pour un ou deux ans. La responsabilité de la scolarité obligatoire incombe aux cantons. Les communautés organisent la gestion de l'école. Dans la suite, le terme habituel en Suisse - école élémentaire - est utilisé.

La scuola dell'obbligo in Svizzera, è composta dalla scuola primaria e secondaria inferiore. Nella maggior parte dei cantoni, l'iscrizione all'asilo è obbligatoria per uno o due anni. I cantoni sono le autorità responsabili dell'istruzione obbligatoria. Le comunità organizzano la gestione della scuola.

Compulsory schooling in Switzerland includes primary and lower secondary education. In most cantons, attendance of kindergarten is compulsory for one or two years. Compulsory education is largely rooted in the cantons and local municipalities.

\section{Literatur}

Al-Anshory, A. S. \& Ali, H. M. (2014). The gap between primay and secondary school teacher followership styles at Adni Islamic school, Malaysia. IIUM Journal of Educational Studies, 2, (2), 59-83.

Altrichter, H., Rürup, M. \& Schuchart, C. (2016). Schulautonomie und die Folgen. In H. Altrichter \& K. Maag Merki (Hrsg.), Handbuch Neue Steuerung im Schulsystem (S. 107-149). Wiesbaden, Deutschland: Springer Fachmedien Wiesbaden.

Baker, S. D. (2007). Followership: The theoretical foundation of a contemporary construct. Journal of Leadership \& Organizational Studies, 14, (1), 50-60.

Bonsen, M. (2016). Schulleitung und Führung in der Schule. In H. Altrichter \& K. Maag Merki (Hrsg.), Handbuch Neue Steuerung im Schulsystem (S. 301-323). Wiesbaden, Deutschland: Springer Fachmedien Wiesbaden.

Bonsen, M. \& Rolff, H. (2006). Professional learning communities of teachers. Zeitschrift für Pädagogik, 52, (2), 167-184.

Büeler, X. \& Imgrüth, P. (2003). Netzwerk «Schulen mit Profil» [Luzern]. Peer review extended (PRE) (Bericht 2003). Schulen mit Profil, Luzern. Zugriff unter https://edudoc.ch/ record/29279/files/146.pdf

Buhren, C. G., \& Rolff, H.-G. (2002). Personalentwicklung in Schulen: Konzepte, Praxisbausteine, Methoden. Weinheim, Deutschland: Beltz.

Carsten, M. K., Uhl-Bien, M., West, B. J., Patera, J. L. \& McGregor, R. (2010). Exploring social constructions of followership: A qualitative study. The Leadership Quarterly, 21, (3), 543-562.

Cervone, L. \& Martinez-Miller, P. (2007). Classroom walkthroughs as a catalyst for school improvement. Leadership Compass, 4, (4), 1-4.

Clarke, M., Killeavy, M. \& Ferris, R. (2015). Mentor teachers as leaders and followers in school-based contexts in the Republic of Ireland. International Journal of Educational Management, 29, (3), 368-379.

Crippen, C. (2012a). Enhancing authentic leadership-followership: Strengthening school relationships. Management in Education, 26, (4), 192-198.

Crippen, C. (2012b). The importance of followership in schools: First, teacher awareness. Education Canada, 52, (2).

Crossman, B. \& Crossman, J. (2011). Conceptualising followership-a review of the literature. Leadership, 7, (4), 481-497.

Day, C., Elliot, B. \& Kington, A. (2005). Reform, standards and teacher identity: Challenges of sustaining commitment. Teaching and Teacher Education, 21, (5), 563-577.

Densten, 1. L. \& Gray, J. H. (2001). The Links between followership and the experiential learning model: Followership coming of age. Journal of Leadership \& Organizational Studies, 8, (1), 69-76. 
Departement Bildung, Kultur und Sport. (2006). Geleitete Schule. Einrichten einer Schulleitung: Informationen. Aarau, Schweiz: Departement Bildung, Kultur und Sport (Kanton Aargau).

EDK (Schweizerische Konferenz der kantonalen Erziehungsdirektoren). (2007). Aufbau des Bildungssystems und Bildungsverwaltung. Kapitel 2 des Schweizer Beitrags für die Datenbank "Eurybase-The Database on Education Systems in Europe». Verfügbar unter http://www. edk.ch/dyn/bin/12961-13431-1-eurydice_02d.pdf

Eikenbusch, G. (2002). Didaktische Kompetenzen im Lehrerberuf heute. Professionalität gemeinsam erarbeiten. Pädagogik (Weinheim), 54, (11), 13-16.

Field, A. (2013). Discovering statistics using IBM SPSS statistics. London, England: Sage.

Goldhorn, J., Kearney, W. S., \& Webb, M. (2013). Classroom walkthrough practices: lessons learned from 10,000 observations. National Forum of Educational Administration \& Supervision Journal, 30(3), 20-28.

Gruber, H. \& Leutner, D. (2003). Die kompetente Lehrperson als Multiplikator von Innovation. In I. Gogolin \& R. Tippelt (Hrsg.), Innovation durch Bildung: Beiträge zum 18. Kongress der Deutschen Gesellschaft für Erziehungswissenschaft (S. 263-274). Wiesbaden, Deutschland: VS Verlag für Sozialwissenschaften.

Harazd, B., Gieske, M. \& Gerick, J. (2012). Was fördert affektives Commitment von Lehrkräften? Eine Analyse individueller und schulischer (Bedingungs-)Faktoren. Zeitschrift für Bildungsforschung, 2, (2), 151-168.

Heck, R. H. \& Hallinger, P. (2009). Assessing the contribution of distributed leadership to school improvement and growth in Math achievement. American Educational Research Journal, 46, (3), 659-689.

Henkin, A. B. \& Holliman, S. L. (2009). Urban teacher commitment. Urban Education, 44, (2), $160-180$.

Hinić, D., Grubor, J. \& Brulić, L. (2016). Followership styles and job satisfaction in secondary school teachers in Serbia. Educational Management Administration \& Leadership, 45, (3), 503-520.

Hof, S., Strupler, M. \& Wolter, S. C. (2011). Quereinsteiger in den Lehrberuf am Beispiel der schweizerischen Berufsbildung. Bern, Schweiz: Swiss Leading House Economics of Education, Firm Behavior and Training Policies.

Holtappels, H. G., Lossen, K., Spillebeen, L. \& Tillmann, K. (2011). Schulentwicklung und Lehrerkooperation in Ganztagsschulen. Zeitschrift für Erziehungswissenschaft, 14, (3), 25.

Hooff, B. v. d. \& Ridder, J. A. d. (2004). Knowledge sharing in context: the influence of organizational commitment, communication climate and CMC use on knowledge sharing. Journal of Knowledge Management, 8, (6), 117-130.

Kelley, R. E. (1988). In praise of followers. Harvard Business Review Case Services, (November-December), 8-142.

Kelley, R. E. (1992). The Power of Followership: How to Create Leaders People Want to Follow, and Followers who Lead Themselves. New York, NY: Doubleday/Currency.

Krüger, M. \& Scheerens, J. (2012). Conceptual Perspectives on School Leadership. In J. Scheerens (Hrsg.), School Leadership Effects Revisited: Review and Meta-Analysis of Empirical Studies (S. 1-30). Dordrecht, Netherlands: Springer Netherlands.

Küpers, W. \& Weibler, J. (2008). Inter-leadership: Why and how should we think of leadership and followership integrally? Leadership, 4, (4), 443-475.

LCH (Dachverband Schweizer Lehrerinnen). (2008, Juni). Berufsleitbild und Standesregeln. Paper presented at the LCH-Delegiertenversammlung, Zürich.

Leutwyler, B. (2003). Geleitete Volksschulen im Kanton Schwyz. Zürich: Forschungsbereich Schulqualität \& Schulentwicklung, Pädagogisches Institut, Universität Zürich.

Leutwyler, B. \& Herzog, S. (2010). Zwischen polyvalentem Spezialist und spezialisiertem Generalist: Implikationen für den Lehrberuf. In A. Abächerli (Hrsg.), Entwicklungs- 
landkarte für Lehrpersonen: Notwendigkeit, Konzept und Implikationen eines biografisch orientierten Personalentwicklungsinstruments (S. 135-149). Bern, Schweiz: Haupt Verlag AG.

Maag Merki, K., \& Steinert, B. (2006). Die Prozessstruktur von teilautonomen Schulen und ihre Effektivität für die Herstellung optimaler Lernkontexte für schulische Bildungsprozesse. Schweizerische Zeitschrift für Bildungswissenschaften, 28, 81-100.

Mahler, S. \& Quesel, C. (2015). Die externe Schulevaluation in der Schweiz. Stand und Perspektiven. Journal für Schulentwicklung, 19, (3), 20-25.

Mathieu, J. E. \& Zajac, D. M. (1990). A review and meta-analysis of the antecedents, correlates, and consequences of organizational commitment. Psychological bulletin, 108, (2), 171.

Mertler, C. A., Steyer, S. \& Petersen, G. J. (1997, Oktober). Teachers' perceptions of the leadership/followership dialectic. Paper presented at the Annual Meeting of the Midwestern Educational Research Association, Chicago, IL.

Muijs, D. \& Harris, A. (2003). Teacher leadership-Improvement through empowerment? Educational Management \& Administration, 31, (4), 437-448.

Muijs, D. \& Harris, A. (2006). Teacher led school improvement: Teacher leadership in the UK. Teaching and Teacher Education, 22, (8), 961-972.

Pelz, W. (2016). Transformationale Führung - Forschungsstand und Umsetzung in der Praxis. In C. von Au (Hrsg.), Wirksame und nachhaltige Führungsansätze: System, Beziehung, Haltung und Individualität (S. 93-112). Wiesbaden, Deutschland: Springer Fachmedien Wiesbaden.

Poekert, P. E. (2012). Teacher leadership and professional development: Examining links between two concepts central to school improvement. Professional Development in Education, 38, (2), 169-188.

Pont, B., Nusche, D. \& Moorman, H. (2008). Improving school leadership. Volume 1: Policy and practice (Vol. 1). Paris, France: OECD.

Prince, O. (2004). 10 Jahre SmP - Vom vagen Projekt zur Implementierung. FS\&S aktuell, $1,7$.

Protheroe, N. (2009). Using classroom walkthroughs to improve instruction. Principal, 88, (4), 30-34.

Riketta, M. (2002). Attitudinal organizational commitment and job performance: A meta-analysis. Journal of Organizational Behavior, 23, (3), 257-266.

Robinson, V. M. (2009). Fit for purpose: An educationally relevant account of distributed leadership. In A. Harris (Hrsg.), Distributed Leadership: Different Perspectives (S. 219-240). Dordrecht, Netherlands: Springer Netherlands.

Robinson, V. M., Lloyd, C. A. \& Rowe, K. J. (2008). The impact of leadership on student outcomes: An analysis of the differential effects of leadership types. Educational administration quarterly, 44, (5), 635-674.

Schweinberger, K. \& Huber, B. (in Druck). Aktiv, verbindlich und kooperativ - Lehrpersonen als Mitarbeitende. In C. Quesel \& N. Safi (Hrsg.), Schulentwicklung im Spannungsfeld von Daten und Taten. Standortbestimmungen und Perspektiven in der Schweiz (S.). Bern, Schweiz: hep Bildungsverlag.

Sergiovanni, T. J. (1992). Moral leadership: Getting to the heart of school improvement. San Francisco, CA: Jossey-Bass.

Sergiovanni, T. J. (1998). Leadership as pedagogy, capital development and school effectiveness. International Journal of Leadership in Education, 1, (1), 37-46.

Somech, A. \& Bogler, R. (2002). Antecedents and consequences of teacher organizational and professional commitment. Educational Administration Quarterly, 38, (4), 555-577.

Steger Vogt, E. \& Appius, S. (2011). Personalentwicklung als Führungsaufgabe von Schulleitungen (Bericht zur Befragung von Schulleitungen Kanton St.Gallen). St.Gallen, Schweiz: Pädagogische Hochschule St. Gallen. 
Steiner, P. \& Landwehr, N. (mit Bucher, A., Gsell, M., Gut, M. \& Schweinberger, K.) (2015). Monitoringbericht externe Schulevaluation (Bericht über die Resultate der externen Schulevaluationen der Aargauer Volksschulen zuhanden des Departementes Bildung, Kultur und Sport). Fachhochschule Nordwestschweiz Pädagogische Hochschule.

Steinert, B. \& Maag Merki, K. (2009). Kooperation zwischen Lehrpersonen und Schulen. Empirische Analysen und offene Forschungsfragen. Beiträge zur Lehrerbildung, 27, (3), 395-403.

Terhart, E. (2016). Personalauswahl, Personaleinsatz und Personalentwicklung an Schulen. In H. Altrichter \& K. Maag Merki (Hrsg.), Handbuch Neue Steuerung im Schulsystem (S. 279-299). Wiesbaden, Deutschland: Springer Fachmedien Wiesbaden.

Terhart, E. \& Klieme, E. (2006). Kooperation im Lehrerberuf: Forschungsproblem und Gestaltungsaufgabe. Zur Einführung in den Thementeil. Zeitschrift für Pädagogik, 52, (2), 163-166.

Uhl-Bien, M., Riggio, R. E., Lowe, K. B. \& Carsten, M. K. (2014). Followership theory: A review and research agenda. The Leadership Quarterly, 25, (1), 83-104.

Van Dick, R., Hirst, G., Grojean, M. W. \& Wieseke, J. (2007). Relationships between leader and follower organizational identification and implications for follower attitudes and behaviour. Journal of Occupational and Organizational Psychology, 80, (1), 133-150.

van Quaquebeke, N. \& Brodbeck, F. C. (2008). Entwicklung und erste Validierung zweier Instrumente zur Erfassung von Führungskräfte-Kategorisierung im deutschsprachigen Raum. Zeitschrift für Arbeits- und Organisationspsychologie, 52, (2), 70-80.

Schlagworte: Followership, obligatorische Schule, berufsbegleitende Schule

\section{La «Followership» dans le domaine de l'éducation exige de l'expérience. Comparaison entre les écoles obligatoires et les écoles professionnelles}

\section{Résumé}

La plus grande liberté de conception et l'autonomisation partielle des écoles élémentaires va de pair avec une plus grande responsabilité de la qualité de l'éducation et de l'enseignement. Ici, les enseignants jouent un rôle central: en tant qu'employés, ils doivent montrer de l'engagement envers les objectifs de l'école et les décisions prises par la direction scolaire, prendre activement la responsabilité du développement de l'enseignement et de l'école et coopérer plus étroitement au sein de l'équipe que par le passé. Contrairement aux écoles primaires, les enseignants des écoles professionnelles connaissent mieux le rôle de "follower». D'une part, une direction administrative et organisationnelle est établie depuis longtemps dans les écoles professionnelles, qui sont responsables de la gestion pédagogique, de la gestion du personnel ainsi que du développement de l'école et de la qualité. D'autre part, les enseignants des écoles professionnelles sont tenus d'exercer une profession de base avec une expérience professionnelle correspondante et sont donc familiarisés avec le rôle de «follower» dans ce contexte également. Le présent article examine l'impact de l'expérience variée dans le rôle de «follower» des enseignants des écoles élémentaires respectivement profession- 
nelles quant aux attributs de "follower» autodéclarés, d'une part, et sur l'image de bons employés d'autre part. Dans ce contexte, les attributs suivants sont pris en compte: l'engagement (le "commitment»), la contribution active au développement de l'école et des cours ainsi que la coopération au sein de l'équipe. Les conclusions sur les différences de groupe dans la manifestation autodéclarée de l'engagement et de la coopération ainsi que dans l'image de bons employés et leurs implications pour la pratique scolaire et la recherche sont discutées.

Mots clés: Followership, écoles obligatoires, écoles professionnelles

\section{La "followership» in ambito educativo richiede esperienza. Confronto tra scuole dell'obbligo e scuole professionali}

\section{Riassunto}

La maggiore libertà di progettazione e la parziale autnomia che contraddistingono le scuole elementari vanno di pari passo a una maggiore attenzione alla qualità dell' 'istruzione e dell'insegnamento. In un simile contesto, gli insegnanti svolgono un ruolo chiave: in qualità di dipendenti, essi devono mostrare impegno verso gli obiettivi della scuola e allinearsi alle decisioni della direzione scolastica, assumersi la responsabilità attiva dell'insegnamento ("commitment») e dello sviluppo scolastico e cooperare più strettamente con la propria equipe.

A differenza delle scuole elementari, gli insegnanti delle scuole professionali hanno maggiore familiarità con il ruolo di seguace (follower). Da un lato, le scuole professionali si sono viste introdurre già da tempo una leadership amministrativo-organizzativa responsabile dei contenuti pedagogici, della gestione del personale, dello sviluppo della scuola e della qualità dell'insegnamento. Dall'altro lato, siccome gli insegnanti delle scuole professionali devono avere un'occupazione di base con corrispondente esperienza professionale tendono pertanto ad avere una certa familiarità con il ruolo di seguce.

Il presente articolo esamina l'impatto delle differenti esperienze degli insegnanti delle scuole professionali e della scuola dell'obbligo sugli attributi auto-dichiarati di seguace o follower e sull'immagine di buon dipendente. In tale contesto, vengono presi in considerazione: l'impegno («commitment»), il contributo attivo allo sviluppo della scuola e delle lezioni, nonché la cooperazione all'interno dell'equipe. Il presente articolo si sofferma inoltre sui risultati concernenti le differenze di gruppo nel livello auto-dichiarato di "commitment» e cooperazione, nonché nella immagine del buon dipendente e le loro implicazioni per la pratica scolastica e la ricerca.

Parole chiave: Followership, scuola dell'obbligo, scuole professionali 


\section{Followership in education requires experience. A comparison of compulsory and vocational schools}

\section{Summary}

The greater autonomy granted to schools in the course of their partial self-governance goes hand in hand with greater responsibility for the quality of schools and teaching. Teachers play a central part in this: in their new role as followers, they must demonstrate commitment to the goals of the school and the decisions of the school management, proactively assume responsibility for teaching and school development and cooperate more closely in the team.

Unlike in compulsory schools, teachers at vocational schools are more familiar with the follower role. On the one hand, an administrative-organizational management, which is responsible for pedagogical management, personnel management as well as school and quality development, has already been established at vocational schools for some time. On the other hand, teachers at vocational schools must have a regular occupation with corresponding professional experience and are therefore already familiar with the follower role in this context.

This article examines how the different followership experience of compulsory and vocational school teachers affect the self-reported follower attributes as well as the understanding of a good follower. In this context, the attributes commitment, active contribution to school and classroom development as well as team cooperation are considered.

Findings on group differences in the self-reported degree of commitment and cooperation as well as in the understanding of good followers and their implications for school practice and research are discussed.

Parole chiave: Followership, compulsory schools, vocational schools 